\title{
Contents to Volume 131 (2002)
}

Volume 131 No. 1, March 2002

Sebastian Del Baño: On the Motive of Moduli Spaces of Rank Two Vector Bundles over a Curve

Anna Otwinowska: Composantes de dimension maximale d'un analogue $\mathrm{du}$ lieu de Noether-Lefschetz (Components of Maximal Dimension of an Analogue of the Noether-Lefschetz Locus)

E. Vasserot: On the Action of the Dual Group on the Cohomology of Perverse Sheaves on the Affine Grassmannian

Scott T. Parsell and Trevor D. Wooley: On Pairs of Diagonal Quintic Forms

Yves Laurent et Zoghman Mebkhout: Image inverse d'un $\mathcal{D}$-module et polygone de Newton (Inverse Image of a $\mathcal{D}$-module and Newton Polygon)

\section{Volume 131 No. 2, April 2002}

T. Cooper, D. Mond and R. Wik Atique: Vanishing Topology of Codimension 1 Multi-germs over $\mathbb{R}$ and $\mathbb{C}$

Peter Vermeire: On the Regularity of Powers of Ideal Sheaves

Yu. F. Bilu, B. Brindza, P. Kirschenhofer, Á. Pintér and R. F. Tichy: Diophantine Equations and Bernoulli Polynomials with an Appendix by A. Schinzel

Giovanni Felder, Jürg Fröhlich, Jürgen Fuchs and Christoph Schweigert: Correlation Functions and Boundary Conditions in Rational Conformal Field Theory and Three-Dimensional Topology 
Volume 131 No. 3, May 2002

Masanori Katsurada and Kohji Matsumoto: Explicit Formulas and Asymptotic Expansions for Certain Mean Square of Hurwitz Zeta-Functions: III

Jan Denef and François Loeser: Motivic Integration, Quotient Singularities and the McKay Correspondence

Sándor J. Kovács: Logarithmic Vanishing Theorems and Arakelov-Parshin Boundedness for Singular Varieties

Pietro Corvaja and Umberto Zannier: Some New Applications of the Subspace Theorem

Kay Wingberg: On the Fontaine-Mazur Conjecture for CM-Fields

$341-354$

Author Index, Volume 131 (2002) 